\title{
Phosphorylated proteomics analysis of human coronary artery endothelial cells stimulated by Kawasaki disease patients serum
}

Shui-Ming Li ${ }^{1+}$, Wan-Ting Liu ${ }^{2 \dagger}$, Fang Yang ${ }^{3}$, Qi-Jian Yi ${ }^{5^{*}}$, Shuai Zhang ${ }^{4^{*}}$ and Hong-Ling Jia ${ }^{2^{*}}$ (D)

\begin{abstract}
Background: Kawasaki disease (KD) is an acute febrile childhood systemic vasculitis that disturbs coronary arteries. The pathogenesis remains unknown. The study of phosphorylated proteins helps to elucidate the relevant pathophysiological mechanisms of cardiovascular disease. However, few researches explored phosphorylated proteins in KD patients.

Methods: We compared phosphoprotein profiles of HCAECs stimulated by the serum of KD patients and normal children using iTRAQ technology, $\mathrm{TiO}_{2}$ enrichment phosphorylated peptide and MS analysis. Then we conducted the functional analysis by ClueGO and the biological interaction networking analysis by ReactomeFIViz. Western blotting was performed to identify the hub proteins.

Results: Our results revealed that phosphorylation of 148 proteins showed different intensities between the two HCAECs groups, which are enriched in MAPK, VEGFR, EGFR, Angiopoietin receptor, mTOR, FAK signaling pathway and so on. Through the Network Analyzer analysis, the hub proteins are CDKN1A, MAPK1 and POLR2A, which were experimentally validated.
\end{abstract}

Conclusion: In summary, we provided evidence addressing the valuable phosphorylation signaling that could be useful resource to understand the molecular mechanism and the potential targets for novel therapy of KD.

Keywords: KD, HCAECs, Phosphorylated proteomics, Network analyzer analysis, Hub proteins

\footnotetext{
*Correspondence: qjyi2003@aliyun.com; plum808@yeah.net; jiahongling@aliyun.com

${ }^{+}$Shui-Ming Li and Wan-Ting Liu contributed equally to this work. ${ }^{5}$ Department of Cardiovascular Medicine, Children's Hospital of Chongqing Medical University, Ministry of Education Key Laboratory of Child development and Disorder, China International Science and Technology Coorperation base of Child development and Critical Disorder, Chongqing Key Laboratory of Pediatrics, Chongqing, China

${ }^{4}$ Department of Medical Biochemistry and Molecular Biology, School of Basic Medical Sciences, Jinan University, Guangzhou, Guangdong, China

${ }^{2}$ Key Laboratory of Functional Protein Research of Guangdong Higher Education Institutes, Institute of Life and Health Engineering, College of Life Science and Technology, Jinan University, No.601, West Huangpu Avenue, Guangzhou 510632, Guangdong, China

Full list of author information is available at the end of the article
}

(c) The Author(s). 2019 Open Access This article is distributed under the terms of the Creative Commons Attribution 4.0 International License (http://creativecommons.org/licenses/by/4.0/), which permits unrestricted use, distribution, and reproduction in any medium, provided you give appropriate credit to the original author(s) and the source, provide a link to the Creative Commons license, and indicate if changes were made. The Creative Commons Public Domain Dedication waiver (http://creativecommons.org/publicdomain/zero/1.0/) applies to the data made available in this article, unless otherwise stated. 


\section{Background}

Kawasaki disease (KD) is an immune-related multisystem vasculitis and usually occurs in children under 5 years of age, but whose etiology remains unknown. As the first pathogeny of acquired heart disease instead of rheumatic fever, KD is a novel risk factor of forming atherosclerosis and ischemic heart disease that have been reported by more and more previous studies. The acute vasculitis associated KD may lead to the development of a complex set of coronary artery abnormalities including coronary artery dilatation and coronary aneurysm. And coronary artery stenosis or thrombosis, or even myocardial infarction could occur in later period. However, the pathomechanism of coronary artery abnormalities complicated with KD is still not well understood, making specific molecular diagnosis and therapy thus far impossible.

Protein phosphorylation is one of the most basic, common and important mechanisms for regulating protein activation and function. A variety of biological processes are closely related to the protein phosphorylation which play on/off regulatory role for many biochemical functions, such as transcriptional and translational regulation, signal transduction, DNA damage repair, cell metabolism, secretion, homeostasis and so on. Abnormal phosphorylation associated with many diseases, and the production of phosphorylation is as the footprints of the abnormal status which exists in organisms. Therefore, the study of phosphorylated proteins in biological samples helps to elucidate the relevant pathophysiological mechanisms. Many studies have reported that phosphorylation of proteins play an important regulatory role in the occurrence and development of cardiovascular disease $[1,2]$. Some protein kinases affect the structure and function of vascular smooth muscle cells by mutual regulation with ANG II, PDGF, ET-1 and other vasoactive substances, and therefore directly involved in the pathological process of cardiovascular disease. There have been some previous proteomics studies on KD in serum and urine [3-5]. However, there are few protein phosphorylation studies in field of KD.

Hence, this study focused on KD protein phosphorylation to identify differentially expressed phosphoproteins and phosphorylated molecules in KD by large-scale high-throughput approaches: the isobaric tags for relative and absolute quantitation (iTRAQ) technology, titanium dioxide $\left(\mathrm{TiO}_{2}\right)$ enrichment phosphorylated peptides, mass spectrometry(MS) analysis [6] to investigate the phosphoproteome analysis of KD patients, and revealed that the phosphoprotein plays an important role in the pathogenesis of KD. The study may provide an insightful understanding of KD precise pathomechanism and implications for the therapy of KD in phosphorylation protein level.

\section{Methods}

Collection of serum samples of KD patients and healthy children

Ethical approval was obtained for children with KD and healthy children clinical sample collection from the Ethics Committee at First Affiliated Hospital of Jinan University and written informed consent was obtained from the guardians of all children. Serum samples from children with KD diagnosed were randomly selected according to the revised digest version of guidelines from the Japanese Circulation Society Joint Working Groups performed in 2012 [7]. The information of the children with KD is shown in Table 1 . Serum samples from sex and age matched normal children were used as the control group. Serum aliquots were collected and stored at $-80^{\circ} \mathrm{C}$ refrigerator.

Human coronary artery endothelial cells (HCAECs) culture and preparation

HCAECs (human coronary artery endothelial cells) were obtained from ScienCell (Carlsbad, CA, USA) and cultured using an endothelial cell growth medium containing growth factors, supplements and 10\% fetal bovine serum. When HCAECs were $90 \%$ confluent, the medium was exchanged to endothelial cell basal medium and the cells were incubated with $15 \%$ serum from KD patients or healthy children respectively. After culturing of HCAECs for $24 \mathrm{~h}$, two HCAECs groups (Control and KD) were collected.

\section{Protein preparation and ITRAQ labeling}

Control and KD groups were washed three times with ice-cold washing buffer $(10 \mu \mathrm{M}$ Tris- $\mathrm{HCl}, 250 \mu \mathrm{M}$ sucrose, $\mathrm{pH} 7.0$ ) and transferred to a clean $1.5 \mathrm{ml}$ Eppendorf tube. Cells were lysed with a buffer containing $7 \mathrm{M}$ urea, $2 \mathrm{M}$ thiourea, $4 \%$ CHAPS, $0.2 \mathrm{mg} / \mathrm{ml}$ PMSF, phosphatase inhibitors cocktail (Roche, Basel, Switzerland) and protease inhibitors (Roche, Basel, Switzerland). Cellular debris was removed by centrifugation for 30 min at $13,000 \mathrm{~g}$ and at $4{ }^{\circ} \mathrm{C}$. Protein concentration was determined by Protein Assay Kit. The total proteins of

Table 1 Clinical indicators of 8 KD patients

\begin{tabular}{llll}
\hline Patient & Age-ranges & Gender & Coronary Change \\
\hline Patient 1 & $2-5 Y$ & male & $\mathrm{LCA}=3.0 \mathrm{~mm}, \mathrm{RCA}=2.6 \mathrm{~mm}$. \\
Patient 2 & $1-2 \mathrm{Y}$ & male & $\mathrm{LCA}=2.8 \mathrm{~mm}, \mathrm{RCA}=2.5 \mathrm{~mm}$. \\
Patient 3 & $2-5 \mathrm{Y}$ & male & $\mathrm{LCA}=4.0 \mathrm{~mm}, \mathrm{RCA}=3.6 \mathrm{~mm}$. \\
Patient 4 & $2-5 \mathrm{Y}$ & female & $\mathrm{LCA}=3.0 \mathrm{~mm}, \mathrm{RCA}=2.5 \mathrm{~mm}$. \\
Patient 5 & $2-5 \mathrm{Y}$ & male & $\mathrm{LCA}=3.5 \mathrm{~mm}, \mathrm{RCA}=3.6 \mathrm{~mm}$. \\
Patient 6 & $2-5 \mathrm{Y}$ & male & $\mathrm{LCA}=3.2 \mathrm{~mm}, \mathrm{RCA}=2.8 \mathrm{~mm}$. \\
Patient 7 & $2-5 \mathrm{Y}$ & female & $\mathrm{LCA}=3.2 \mathrm{~mm}, \mathrm{RCA}=3.0 \mathrm{~mm}$. \\
Patient 8 & $1-2 \mathrm{Y}$ & female & $\mathrm{LCA}=3.2 \mathrm{~mm}, \mathrm{RCA}=3.3 \mathrm{~mm}$.
\end{tabular}

$Y$ : year; $L C A$ : left coronary artery; $R C A$ : right coronary artery 
each group were analyzed by iTRAQ-based liquid chromatography and tandem mass spectrometry/mass spectrometry (LC-MS/MS). $200 \mu \mathrm{g}$ protein sample from each group was reduced, alkylated, and subjected to tryptic hydrolysis [8]. ITRAQ labeling was performed on the basis of iTRAQ Regents manufacturer's protocol. Each sample was labeled separately with the iTRAQ tags as follows: KD group was labeled with 113/115 isobaric tags;Control group was labeled with $117 / 119$ isobaric tags separately, and then all labeled peptides were pooled and evaporated to dryness in a vacuum centrifuge.

\section{High-pH reversed-phase liquid chromatography}

Firstly, iTRAQ labeled samples were diluted to $100 \mu \mathrm{l}$ with $\mathrm{H}_{2} \mathrm{O}$ buffer $\left(\mathrm{NH}_{3} \cdot \mathrm{H}_{2} \mathrm{O}, \mathrm{pH}=10\right)$ before high performance liquid chromatography (HPLC) on a Gemini-NX 3u C18 110A; $150 \times 2.00 \mathrm{~mm}$ Phenomenex columns and Gemini 3u C6-Phenyl 110A; $100 \times 2.0 \mathrm{~mm}$. The flow rate with 0.2 $\mathrm{ml} / \mathrm{min}$ was used for reversed-phase column separation by $\mathrm{H}_{2} \mathrm{O}$ (mobile phase A) and $80 \%$ acetonitrile (ACN) (mobile phase B). A solvent gradient system was used as described previously [8].

\section{Phosphopeptides enrichment employing $\mathrm{TiO}_{2}$ resin}

Phosphopeptide Enrichment $\mathrm{TiO}_{2}$ kit (Calbiochem, San Diego, CA, USA) was used to enrich the phosphopeptides after peptide digestion according to the manufacturer's instruction with minor modifications. In brief, the production of trypsin digestion was dried and redissolved in $200 \mu \mathrm{TiO}_{2}$ phosphobind buffer with $50 \mathrm{~g} / \mathrm{L} \mathrm{2}$, 5-dihydroxybenzoic acid. Then $50 \mu \mathrm{TiO}_{2}$ phosphobind resin was added and incubated for $30 \mathrm{~min}$. After discarding supernatant, $\mathrm{TiO}_{2}$ was rinsed for three times with the wash buffer. Elute the phosphopeptides with elution buffer for twice and combine all the eluates. And then the eluates were dried using a Speed-Vac concentrator and reconstituted in $2 \% \mathrm{ACN} / 1 \%$ TFA for LC-MS/MS analysis.

\section{Peptides analysis by the LC-MS/MS approach}

Dried phosphopeptides were analyzed with a Triple TOF 5600 plus nano ESI-LCMS instrument. Briefly, the peptide mixtures were loaded in a $\mathrm{C} 18$ column $(5 \mu \mathrm{m}$ resin from Michrom Bioresources, $10 \mathrm{~cm}$ long, $100 \mu \mathrm{m}$ i.d., Auburn, CA, USA) using an autosampler. Peptides were eluted by the $0-35 \%$ gradient buffer solution (Buffer A: $5 \%$ ACN and $0.1 \%$ formic acid; Buffer B: $95 \% \mathrm{ACN}$ and $0.1 \%$ formic acid) for more than $90 \mathrm{~min}$ and subsequently online detected in the Triple TOF 5600 plus mass spectrometer using an information dependent acquition mode (IDA) method which allows top 20 precusor ions selected for MS/MS analysis in each cycle. The general mass spectrometric conditions were as the follows: spray voltage of 2.3 $\mathrm{kV}$; curtain gas of $35 \mathrm{psi}$, nebulizer gas of $5 \mathrm{psi}$, and an interface heat temperature of $150^{\circ} \mathrm{C}$.The MS and MS2 analysis was operated in positive TOF-MS and production ion scan mode respectively. For IDA analysis, survey scans were acquired in $250 \mathrm{~ms}$ and as many as 20 product ion scans $(80 \mathrm{~ms})$ were collected if the precursor ion intensity passed the threshold of $200 \mathrm{cps}$ with the charge state of between of +2 to +5 . A rolling collision energy setting was applied to all precursor ions. Dynamic exlusion was set for $16 \mathrm{~s}$.

Database analysis and manual evaluation of mass spectra The MS/MS data were analyzed for protein identification and quantification using ProteinPilot Software v.4.5 (AB Sciex, Framingham, MA, USA). The local false discovery rate was estimated with the integrated PSPEP tool in the ProteinPilot software to be $1.0 \%$ after searching against a decoy concatenated uniprot human protein database (20,210 protein entries). The database search parameters were as the followings: iTRAQ 8-plex quantification, cysteine modified with iodoacetamide, phosphorylation emphasis, trypsin digestion, thorough searching mode and minimum protein threshold of $95 \%$ confidence (unused protein score $>1.3$ ). The same raw files used for protein pilot 4.5 search analysis were further exported into MGF format peak list files and then submitted to mascot search engineer for protein identification and relative quantitation analysis. The search parameters were the same as those employed in proteinpilot software. Mass spectra of identified phosphopeptides with confidence between 90 and 95\% should also meet the following criteria: two consecutive b- and/or y-ion series and extensive coverage of b- and/ or y-ion series were required, an obvious observation of neutral loss of $98 \mathrm{Da}$ of the precusors.

\section{Western blot analysis}

After lysis, the protein samples of two groups were separated by $12 \%$ SDS-PAGE, electrophoresed and transferred onto a PVDF membrane. Blots were detected with several antibodies CDKN1A (cyclin-dependent kinase inhibitor 1A), MAPK1 (Mitogen-activated protein kinase-1, also known as ERK2 or p42 MAPK), POLR2A (DNA-directed RNA polymerase II subunit RPB1), phospho(p)-CDKN1A (Thr145), p-MAPK1 (Thr202/Tyr204), p-POLR2A (Ser1619) following the standard protocol. CDKN1A, MAPK1, p-CDKN1A (Thr145) and p-MAPK1 (Thr202/ Tyr204) antibodies were purchased from Abcam Inc., USA and other antibodies including POLR2A and p-POLR2A (Ser1619) were purchased from Novus Biologicals Inc., USA. All immunoblot detections were performed with horseradish peroxidase-conjugated secondary antibodies and chemiluminescence detection system. The quantitative analysis of band intensities was performed using Photoshop software. 


\section{Protein categorization and protein-protein interaction} network constructions

The Cytoscape plugin GlueGO and ReactomeFIViz were applied for the differentially expressed phosphoproteins to process the biological process of GO term and KEGG pathway enrichment analysis. The $p$-value $<0.05$ and FDR $<0.01$ were as threshold values for the GO and KEGG pathway enrichment. ReactomeFIViz also provides the associations of these differential expressed proteins which were visualized by network illustration. Then, the betweenness centrality and the number of direct edges were analyzed by Cytoscape plug-in tool 'Network Analyzer', and the analysis results were showed by progressive changed node sizes and colors.

\section{Results}

\section{Quantitative phosphoproteomics analysis of HCAECs of} KD

To obtain a global view change of protein phosphorylation in $\mathrm{KD}$, we simulated HCAECs with serum from KD patients to mimic microenvironment of $\mathrm{KD}$
[9-11]. All MS/MS spectra were respectively searched, against the reversed and forward human protein sequence database to estimate rates of false-positive matches after LC-MS/MS analysis on the enriched phosphopeptides. The 5929 phosphopeptides from the target database passed our criteria. The false positive rate of phosphopeptide was hence estimated to be $1.3 \%$. Multiple filtering standard were established to test and verify search results. For each of the identified phosphorylated peptides in this work, peptide sequences were manually confirmed. We detected 238 unique phosphorylation sites from 233 unique phosphopeptides corresponding to 148 protein groups including the distributions of phosphorylated threonine, serine, and tyrosine sites, respectively, after these validations. This whole dataset was provided as Additional file 1: Table S1. These results are in line with those from a previous study on a variety of cell types: the overall level of phosphotyrosine in proteins was very low compared to the level of phosphoserine and phosphothreonine in HCAECs of KD.

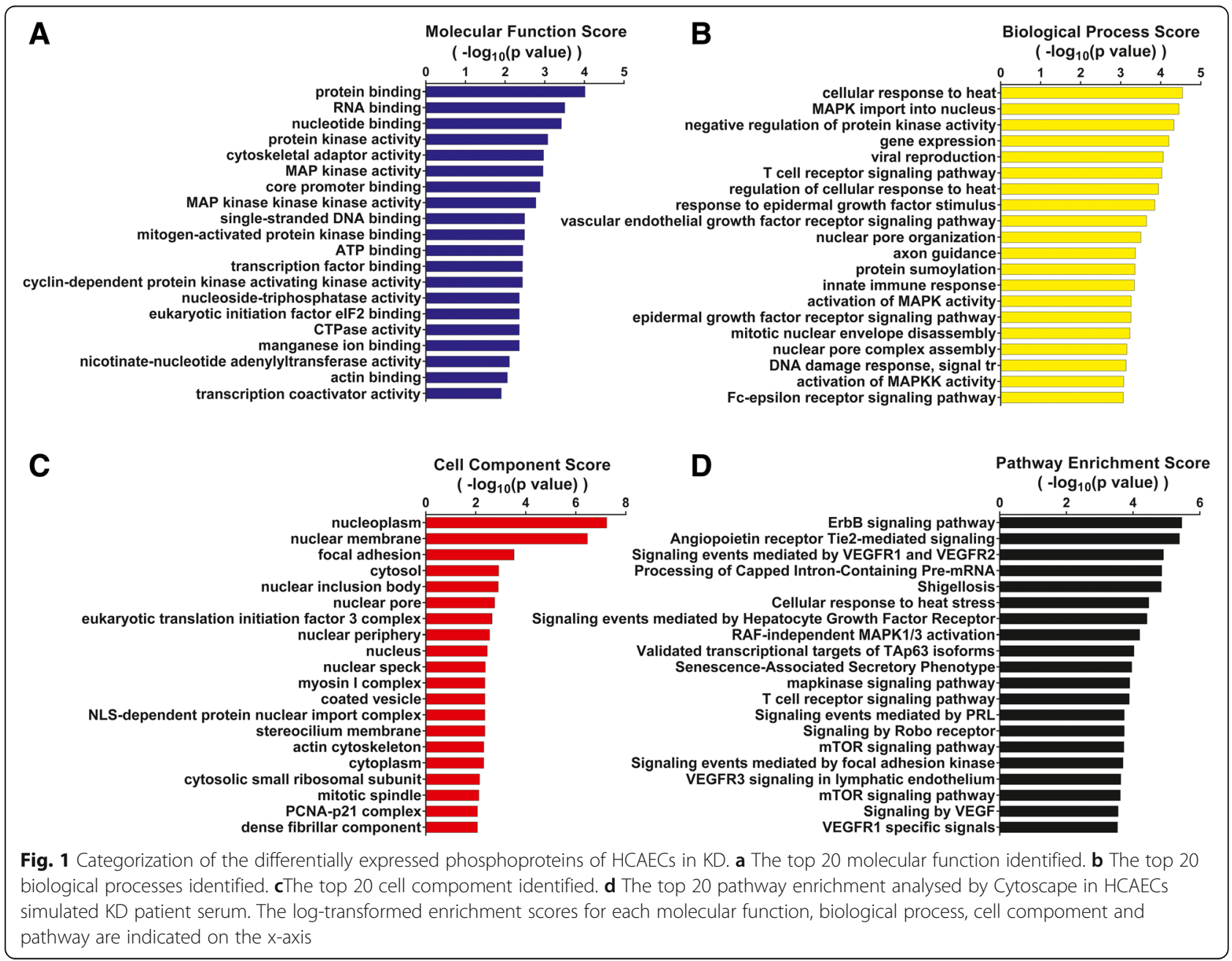




\section{Functional analysis of identified phosphoproteins of HCAECs of KD}

In order to understand the biological relevance of phosphoproteins, the molecular functions, biological processes and protein classification of the differentially expressed proteins were analysed using ClueGO that is a Cytoscape plug in software, which integrates gene ontology terms. Bar charts were used to show the distribution of biological process, functional categories for these differentially expressed proteins of HCAECs in KD. Figure 1 provides an overview of KD phosphoproteome based on the known or postulated functions or biological processes of the identified phosphoproteins.

\section{Biological interaction networking of identified phosphoproteins}

To comprehensively characterize the relationships among significantly differentially expressed phosphoproteins of HCAECs in KD, we were subjected to the KEGG pathway enrichment analysis and signalling network modelling by the Cytoscape plugin ReactomeFIViz. The thresholds of pathway enrichment were defined as $p<0.05, \mathrm{FDR}<1 \%$. We constructed a biological interaction networking of the phosphoproteins identified of HCAECs in KD. The phosphoproteins that could be networked were linked by various relationships such as protein-protein interactions, modifications and regulation of expression. The network was deeply analyzed by Network Analyzer which is a Cytoscape plug-in tool for betweenness centrality and number of direct edges shown in Figs. 2 and 3, the progress changed colours and node sizes showed which nodes play core roles in the whole network, viz. the more betweenness the bigger of node sizes, the more number of direct edges the darker red colour. In other words, the node with the deepest red and the biggest size was the most important core. According to this rule, CDKN1A, MAPK1 and POLR2A were selected.

\section{Validation of differential expressed phosphoproteins}

To further confirm the results from the quantitative phosphoproteome analysis, we chose CDKN1A, MAPK1, POLR2A, p-CDKN1A, p-MAPK1, p-POLR2A, for Western blotting verification using an anti-CDKN1A antibody, anti-MAPK1 antibody, anti-POLR2A antibody, anti-pCDKN1A (Thr145) antibody, anti-p-MAPK1 (Thr202/ Tyr204) antibody and anti-p-POLR2A (Ser1619) antibody. As shown in Fig. 4, the results of Western blotting were consistent with phosphoproteomics analysis for these differentially expressed proteins. Phosphorylation of CDKN1A, MAPK1 and POLR2A are significantly increased in

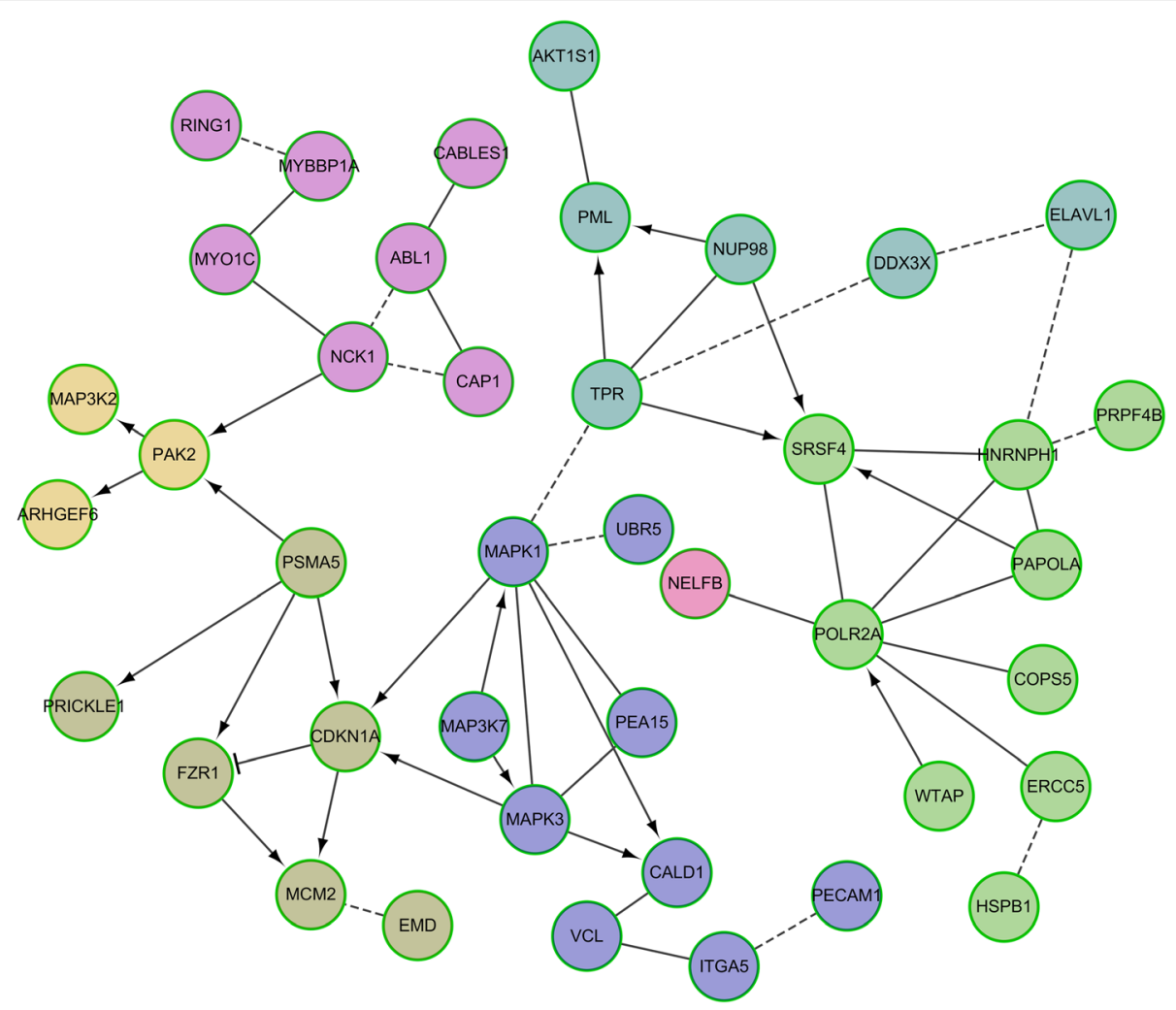

Fig. 2 Biological interaction networking of identified HCAECs phosphoproteins in KD. Proteins of the network were differentially expressed phosphoproteins of HCAECs of KD which were functionally enriched based on KEGG pathway 


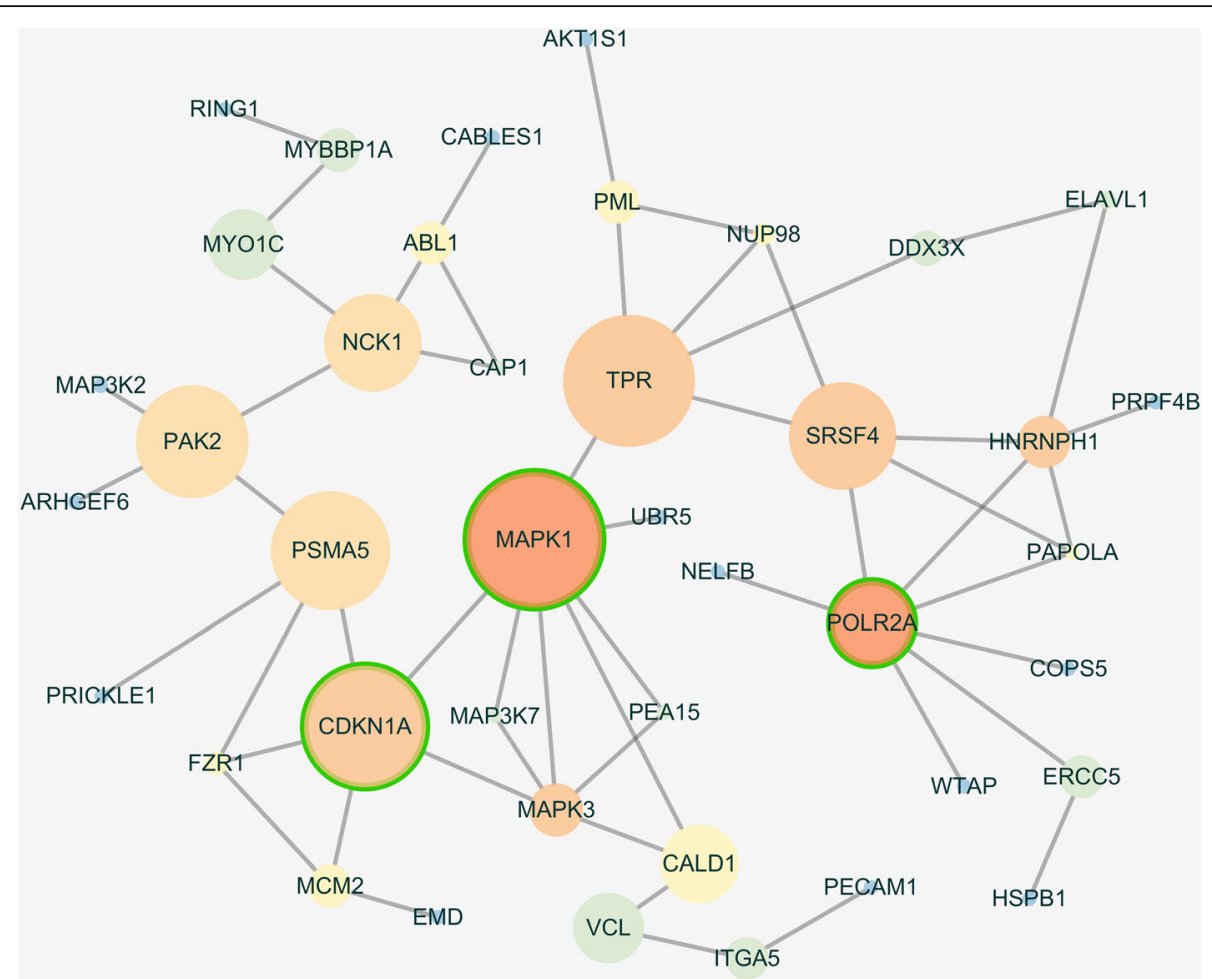

Fig. 3 The outcome of network analysis. The node sizes showed the results of betweenness centrality analysis. The node colour was decided by the numbers of direct edges to indicate the important of the node. The node with green circle demonstrated the proteins were selected for western blotting verification because they contained the best results of the analysis

HCAECs of KD, whereas steady-state CDKN1A, MAPK1 and POLR2A remained almost unchanged in Western blotting verification.

\section{Discussion}

To date, little study has been carried out on the phosphoproteome in KD. To explore pathophysiology-related molecules from the aspect of phosphorylation in $\mathrm{KD}$, we compared phosphoprotein profiles of HCAECs of KD and normal children. Among the detected 5929 phosphopeptides, 233 phosphopeptides corresponding to 148 protein groups showed different intensities between the two HCAECs groups. The identified differentially expressed phosphoproteins may be used as potential biomarkers to facilitate $\mathrm{KD}$ diagnosis and monitoring of treatment effectiveness and it would reflect a deeper understanding of pathological processes of coronary artery abnormalities complicated with KD.

Applying various analysis software tools, we identify several differentially regulated signaling pathways, such as MAPK (mitogen-activated protein kinase), VEGFR (vascular endothelial growth factor receptor), EGFR (epidermal growth factor receptor), Angiopoietin receptor, HGFR (hepatocyte growth factor receptor), mTOR (mammalian target of rapamycin), FAK (focal adhesion kinase), PRL signaling pathway. Some previous study have revealed that AMPK-mTOR and MAPK-ERK1/2 signaling pathway are involved in human vascular smooth muscle cells proliferation, which plays a key role in the pathogenesis of vascular diseases such as hypertension and restenosis [12-15]. In addition, FAK signaling and enhanced tyrosine phosphorylation is important for the human coronary artery smooth muscle cells and cardiac microvascular endothelial cells migration, which is the key process in the pathophysiology of restenosis and atherosclerosis $[16,17]$. Previous studies have noted that the activated FAK, ERK, JNK, PI3K and AKT may promote angiogenesis and arteriogenesis, which is reported to be the mature form of new vessels and lead to an efficient restoration of blood flow [18].

Strikingly for this study, phosphopeptides from proteins including CDKN1A, MAPK1 and POLR2A were remarkably increased expression in KD. CDKN1A (also known as p21) regulates various biological activities by binding to and inhibiting the kinase activity of the CDKs (cyclin-dependent kinases, CDK2 and CDK1 also known as CDC2) leading to cell cycle arrest at specifics tags. Extensive reports in biochemistry and genetics shows that p21 is identified as an oncogene or tumor suppressor due to its up-regulation or down-regulation in several cancers $[19,20]$. In addition, p21 stimulates cell proliferation of endothelial cell depending on 


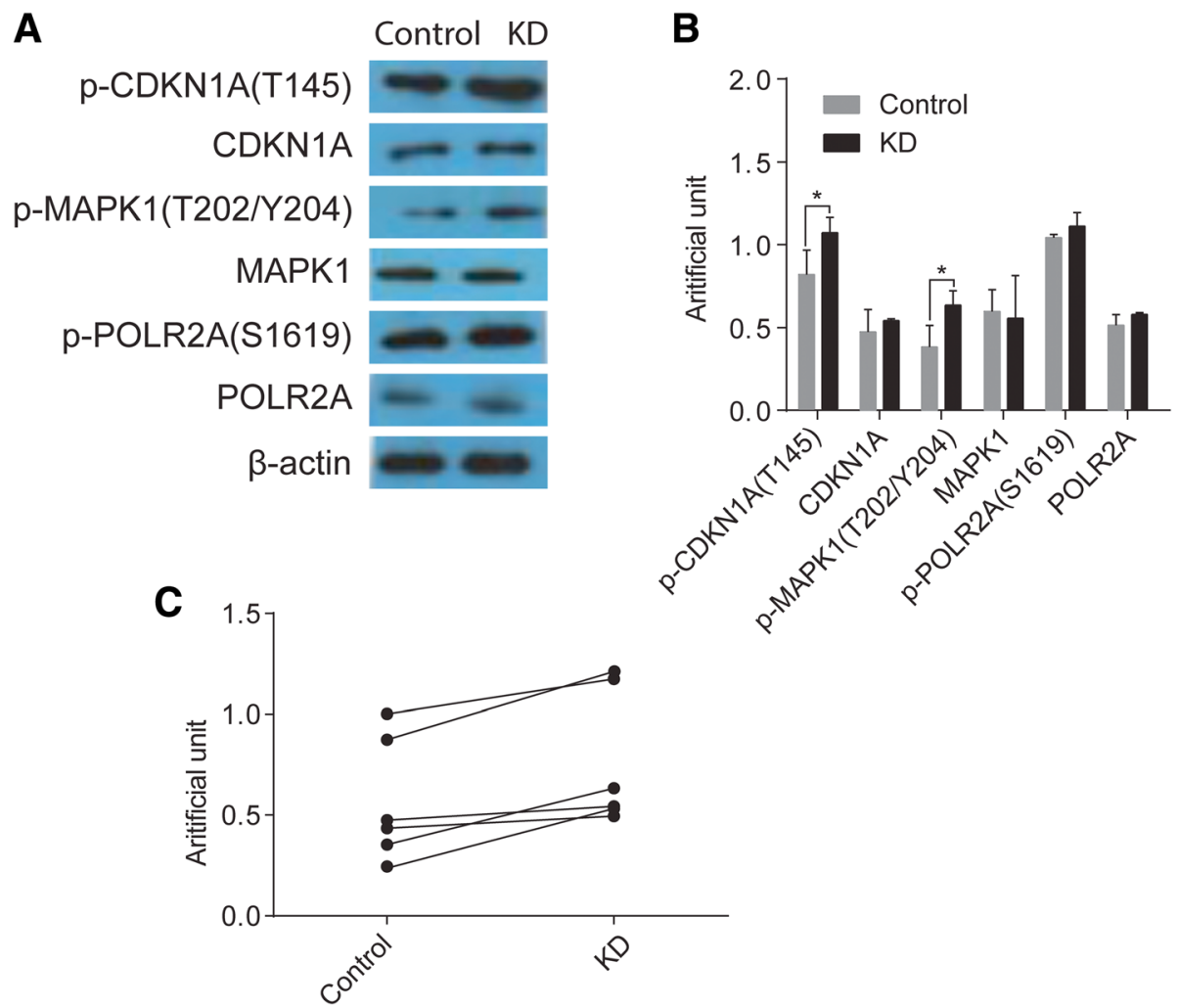

Fig. 4 Validation of phosphorylated proteomics results by Western blot. a Western blots of CDKN1A, MAPK1, POLR2A, p-CDKN1A, p-MAPK1, p-POLR2A of HCAECs in KD, $\beta$-actin was used as the internal control. $\mathbf{b}$ Statistical analysis of the band intensities in A. $\mathbf{c}$ Grouped analysis of the band intensities of A. Data represent mean \pm SD. Statistical significance is determined by Student's $t$ test, $p<0.05$

attenuating CDK2 inhibition which is mediated by AKT1- phosphorylated p21 at T145 [21]. Furthermore, the phosphorylation of p21 by AKT1 in endothelial cells may have a role in promoting neovascularization and metastasis. Interestingly, our results showed that p-p21 (T145) was enhanced in KD, suggested that p21 phosphorylation may have an important role in coronary artery abnormalities of KD.

We are also specifically interested in MAPK1 that plays a pivotal role in cell development, proliferation, differentiation, transcription regulation. The activation of MAPK1 requires its phosphorylation on Tyr and Thr residues by upstream kinases, such as MEK2. Upon activation, MAPK1 translocates to the nucleus of the stimulated cells, where it phosphorylates nuclear targets. The previous studies have discovered that the ERK1/2 activation is able to protect cardiomyocytes against apoptosis [22]. Furthermore, the phosphorylation of MAPK1 can increase the proliferation of cardiomyocytes via up-regulating the expression of MALAT1 through PI3K/ AKT signaling pathway [23]. The MAPK1 genetic mutations were speculated to be potential risk factors for heart defects, such as coronary artery disease considering hereditary variation among diverse ethnicities
[24, 25]. Miura et al. reported the effect of MAPK activation on HDL-mediated signal transduction and angiogenesis induction in HCAECs [26]. And Plasma $\mathrm{C}$-reactive protein, a prototypic marker of inflammation, regulated the expression of receptor for advanced glycation end-products via activation of the ERK/NF- $\kappa B$ signaling pathway in HCAECs [27]. However, there were few reports about the research on MAPK1 in coronary artery lesion of KD. Our data suggested that MAPK1 is probably important target of signaling pathways for the development and progression of KD.

The third protein we specifically considered is POLR2A (also known as RPB1), which contains a carboxy terminal domain composed of heptapeptide repeats that are essential for polymerase activity. These repeats contain serine and threonine residues that are phosphorylated in actively transcribing RNA polymerase. POLR2A gene located in close proximity to the tumor suppressor gene p53, which frequently shows loss of heterozyosity in cancer cells. Jesper V. Olsen et al. found that the kinase CDK7 phosphorylates POLR2A and regulates epithelial ovarian cancer cell proliferation in order to reveal a druggable kinase signature in ovarian cancer by 
phosphoproteomics [28]. Change of POLR2A phosphorylation suggest that dysregulation of RNA polymerase may be associated with KD occurrence and development.

\section{Conclusions}

In sum, the in vivo quantitative phosphoproteomics from KD patient serum stimulated HCAECs could lead to provide valuable clues for decreasing the incidence of coronary artery lesions and improving the prognosis in KD. The study may provide an insightful understanding of $\mathrm{KD}$ precise pathomechanism and implications for the treatment of KD in protein phosphorylation level.

\section{Additional file}

Additional file 1: Table S1. Phosphorylated proteins identified in HCAECS of KD, The entire dataset of 233 identified unique phosphopeptides. (XLSX $37 \mathrm{~kb})$

\section{Abbreviations}

CDKN1A: Cyclin-dependent kinase inhibitor 1A; EGFR: Epidermal growth factor receptor; FAK: Focal adhesion kinase; HCAECs: Human coronary artery endothelial cells; HGFR: Hepatocyte growth factor receptor; iTRAQ: isobaric tags for relative and absolute quantitation; KD: Kawasaki disease; MAPK1: Mitogen-activated protein kinase-1; MS: Mass spectrometry; mTOR: Mammalian target of rapamycin; POLR2A RNA: Polymerase II subunit $\mathrm{A}_{;} \mathrm{TiO}_{2}$ : Titanium dioxide; VEGFR: Vascular endothelial growth factor receptor

\section{Acknowledgments}

We thank the Guangzhou Women and Children's Medical Center for providing samples and support from the subjects involved in this study.

\section{Funding}

This study was funded by the National Natural Science Foundation of China (81500275 and 81672781), Guangdong Natural Science Foundation (2016A030313080) and the Fundamental Research Funds for the Central Universities (21616323). The funding body provided financial support only (no participation in the design of the study, collection, analysis, or interpretation of data, nor in writing the manuscript).

\section{Availability of data and materials}

The data used in this study are not openly available because providing data to other third-party individuals is not permitted by the Medical Ethics Committee of the Guangzhou Women and Children's Medical Center.

\section{Authors' contributions}

QJY, SML, SZ and HL made substantial contributions to conception and design. FY participated in sample diagnosis and collection. SML contributed to acquisition of data. WTL were responsible for analysis and interpretation of data. QJY, SML, FY and HLJ were involved in drafting the manuscript. HJL, WTL and SZ revised the manuscript critically for important intellectual content. All authors given final approval of the version to be published and agreed to be accountable for all aspects of the work in ensuring that questions related to the accuracy or integrity of any part of the work are appropriately investigated and resolved.

\section{Ethics approval and consent to participate}

This study was approved by the Ethics Committee at Guangzhou Women and Children's Medical Center (trial no. 077 2013). Written informed consent for participation in this study was obtained by each child participants' legal guardian on their behalf. A copy of the written consent is available for review by the Editor of this journal.
Consent for publication

Not applicable.

\section{Competing interests}

The authors declare that they have no competing interests.

\section{Publisher's Note}

Springer Nature remains neutral with regard to jurisdictional claims in published maps and institutional affiliations.

\section{Author details}

${ }^{1}$ College of Life Sciences and Oceanography, Shenzhen Key Laboratory of Microbial Genetic Engineering, Shenzhen University, Shenzhen, Guangdong, China. ${ }^{2}$ Key Laboratory of Functional Protein Research of Guangdong Higher Education Institutes, Institute of Life and Health Engineering, College of Life Science and Technology, Jinan University, No.601, West Huangpu Avenue, Guangzhou 510632, Guangdong, China. ${ }^{3}$ Department of Pediatrics, First Affiliated Hospital of Jinan University, Guangzhou, China. ${ }^{4}$ Department of Medical Biochemistry and Molecular Biology, School of Basic Medical Sciences, Jinan University, Guangzhou, Guangdong, China. ${ }^{5}$ Department of Cardiovascular Medicine, Children's Hospital of Chongqing Medical University, Ministry of Education Key Laboratory of Child development and Disorder, China International Science and Technology Coorperation base of Child development and Critical Disorder, Chongqing Key Laboratory of Pediatrics, Chongqing, China.

Received: 15 April 2018 Accepted: 17 December 2018

Published online: 17 January 2019

\section{References}

1. Kuzmanov U, Guo H, Buchsbaum D, Cosme J, Abbasi C, Isserlin R, Sharma P, Gramolini AO, Emili A. Global phosphoproteomic profiling reveals perturbed signaling in a mouse model of dilated cardiomyopathy. Proc Natl Acad Sci U S A. 2016;113(44):12592-7.

2. Hafver TL, Hodne K, Wanichawan P, Aronsen JM, Dalhus B, Lunde PK, Lunde M, Martinsen M, Enger UH, Fuller W, et al. Protein phosphatase 1C associated with the cardiac sodium calcium exchanger 1 regulates its activity by dephosphorylating serine 68-phosphorylated Phospholemman. J Biol Chem. 2016;291(9):4561-79.

3. Kentsis A, Shulman A, Ahmed S, Brennan E, Monuteaux MC, Lee YH, Lipsett S, Paulo JA, Dedeoglu F, Fuhlbrigge $\mathrm{R}$, et al. Urine proteomics for discovery of improved diagnostic markers of Kawasaki disease. EMBO Mol Med. 2013; 5(2):210-20.

4. Yu HR, Kuo HC, Sheen JM, Wang L, Lin IC, Wang CL, Yang KD. A unique plasma proteomic profiling with imbalanced fibrinogen cascade in patients with Kawasaki disease. Pediatr Allergy Immunol. 2009;20(7):699-707.

5. Zhang L, Jia HL, Huang WM, Liu CW, Hua L, Liu TC, Mao LJ, Xu YF, Li W, Xia $\mathrm{SL}$, et al. Monitoring of the serum proteome in Kawasaki disease patients before and after immunoglobulin therapy. Biochem Biophys Res Commun. 2014:447(1):19-25.

6. Ge F, Xiao CL, Yin XF, Lu CH, Zeng HL, He QY. Phosphoproteomic analysis of primary human multiple myeloma cells. J Proteome. 2010;73(7):1381-90.

7. JCS Joint Working Group. Guidelines fordiagnosis and management of cardiovascular sequelae in Kawasaki disease (JCS2013). Digest version Circ J. 2014;78(10):2521-62

8. Liu W, Liu C, Zhang L, Xie X, Gu X, Sang C, Xu M, Xu W, Jia H. Molecular basis of coronary artery dilation and aneurysms in patients with Kawasaki disease based on differential protein expression. Mol Med Rep. 2017.

9. Ueno K, Ninomiya Y, Hazeki D, Masuda K, Nomura Y, Kawano Y. Disruption of endothelial cell homeostasis plays a key role in the early pathogenesis of coronary artery abnormalities in Kawasaki disease. Sci Rep. 2017;7:43719.

10. He M, Chen Z, Martin M, Zhang J, Sangwung P, Woo B, Tremoulet AH, Shimizu C, Jain MK, Burns JC, et al. miR-483 targeting of CTGF suppresses endothelial-to-mesenchymal transition: therapeutic implications in Kawasaki disease. Circ Res. 2017;120(2):354-65.

11. Chen ZH, Wan GP, Gu XQ. Effect of small interfering RNA on matrix metalloproteinase- 9 expression in vascular endothelial cells stimulated by serum from children with Kawasaki disease. Zhonghua xin xue guan bing za zhi. 2009;37(9):837-40.

12. Hafizi S, Mordi VN, Andersson KM, Chester AH, Yacoub MH. Differential effects of rapamycin, cyclosporine a, and FK506 on human coronary artery 
smooth muscle cell proliferation and signalling. Vasc Pharmacol. 2004;41(45):167-76.

13. Liou SF, Yeh JL, Liang JC, Chiu CC, Lin YT, Chen IJ. Inhibition of mitogenmediated proliferation of rat vascular smooth muscle cells by labedipinedilol-a through PKC and ERK 1/2 pathway. J Cardiovasc Pharmacol. 2004;44(5):539-51.

14. Owens GK, Kumar MS, Wamhoff BR. Molecular regulation of vascular smooth muscle cell differentiation in development and disease. Physiol Rev. 2004;84(3):767-801.

15. Osman I, Segar L. Pioglitazone, a PPARgamma agonist, attenuates PDGFinduced vascular smooth muscle cell proliferation through AMPKdependent and AMPK-independent inhibition of mTOR/p70S6K and ERK signaling. Biochem Pharmacol. 2016;101:54-70

16. Varadarajulu J, Laser M, Hupp M, Wu R, Hauck CR. Targeting of alpha(v) integrins interferes with FAK activation and smooth muscle cell migration and invasion. Biochem Biophys Res Commun. 2005;331(2):404-12.

17. Xia JB, Mao CZ, Chen ZY, Liu GH, Wu HY, Zhou DC, Park KS, Zhao H, Kim SK, Cai DQ, et al. The CXCL10/CXCR3 axis promotes cardiac microvascular endothelial cell migration via the p38/FAK pathway in a proliferationindependent manner. Exp Mol Pathol. 2016;100(2):257-65.

18. Zuo H, Liu Z, Liu X, Yang J, Liu T, Wen S, Zhang XA, Cianflone K, Wang D. CD151 gene delivery after myocardial infarction promotes functional neovascularization and activates FAK signaling. Mol Med. 2009;15(9-10): $307-15$

19. Shen KC, Heng H, Wang Y, Lu S, Liu G, Deng CX, Brooks SC, Wang YA. ATM and p21 cooperate to suppress aneuploidy and subsequent tumor development. Cancer Res. 2005;65(19):8747-53.

20. Gartel AL. Is p21 an oncogene? Mol Cancer Ther. 2006;5(6):1385-6.

21. Rössig L, Jadidi AS, Urbich C, Badorff C, Zeiher AM, Dimmeler S. Aktdependent phosphorylation of p21Cip1 regulates PCNA binding and proliferation of endothelial cells. Mol Cell Biol. 2001;21(16):5644-57.

22. Lips DJ, Bueno OF, Wilkins BJ, Purcell NH, Kaiser RA, Lorenz JN, Voisin L, SabaEl-Leil MK, Meloche S, Pouysségur J. MEK1-ERK2 signaling pathway protects myocardium from ischemic injury in vivo. Circulation. 2004;109(16):1938.

23. Zhao J, Li L, Peng L. MAPK1 up-regulates the expression of MALAT1 to promote the proliferation of cardiomyocytes through PI3KJAKT signaling pathway. Int J Clin Exp Pathol. 2015:8(12):15947-53.

24. Hoefer J, Azam MA, Kroetsch JT, Leongpoi H, Momen MA, Voigtlaenderbolz J, Scherer EQ, Meissner A, Bolz SS, Husain M. Sphingosine-1-phosphatedependent activation of p38 MAPK maintains elevated peripheral resistance in heart failure through increased myogenic vasoconstriction. Circ Res. 2010; 107(7):923-33

25. Guo N, Zhang N, Yan L, Cao X, Wang J, Wang Y. Correlation between genetic polymorphisms within the MAPK1/HIF-1/HO-1 signaling pathway and risk or prognosis of perimenopausal coronary artery disease. Clin Cardiol. 2017.

26. Miura S, Fujino M, Matsuo Y, Kawamura A, Tanigawa H, Nishikawa H, Saku K. High density lipoprotein-induced angiogenesis requires the activation of Ras/MAP kinase in human coronary artery endothelial cells. Arterioscler Thromb Vasc Biol. 2003;23(5):802-8.

27. Zhong Y, Cheng C, Luo Y, Tian C, Yang H, Liu B, Chen M, Chen Y, Liu S. Creactive protein stimulates RAGE expression in human coronary artery endothelial cells in vitro via ROS generation and ERK/NF-|[kappa]|B activation. Acta Pharmacol Sin. 2015;36(4):440.

28. Francavilla C, Lupia M, Tsafou K, Villa A, Kowalczyk K, Jersiechristensen RR, Bertalot G, Confalonieri S, Brunak S, Jensen LJ. Phosphoproteomics of primary cells reveals Druggable kinase signatures in ovarian Cancer. Cell Rep. 2017;18(13):3242-56

Ready to submit your research? Choose BMC and benefit from:

- fast, convenient online submission

- thorough peer review by experienced researchers in your field

- rapid publication on acceptance

- support for research data, including large and complex data types

- gold Open Access which fosters wider collaboration and increased citations

- maximum visibility for your research: over $100 \mathrm{M}$ website views per year

At $\mathrm{BMC}$, research is always in progress.

Learn more biomedcentral.com/submissions 